\title{
School Health: A Novel School Nurse Clinic Surveillance Project in Coastal Georgia
}

\author{
Amanda Feldpausch ${ }^{* 1,2}$, Wendy Smith ${ }^{3}$, Karl Soetebier ${ }^{4}$, Cherie Drenzek ${ }^{5}$ and Marsha \\ Cornell 6
}

${ }^{1}$ CDC/CSTE Applied Epidemiology Fellowship Program, Atlanta, GA, USA; ${ }^{2}$ Acute Disease Epidemiology Section, Georgia Department of Public Health, Atlanta, GA, USA; ${ }^{3}$ Syndromic Surveillance Project, Georgia Department of Public Health, Atlanta, GA, USA; ${ }^{4}$ Health Information Systems Section, Georgia Department of Public Health, Atlanta, GA, USA; ${ }^{5}$ Epidemiology Section, Georgia Department of Public Health, Atlanta, GA, USA; ${ }^{6}$ School Health Services, Effingham County, Springfield, GA, USA

\begin{abstract}
Objective
This project was designed to demonstrate the feasibility of schoolbased nurse clinic visit syndromic surveillance. Additional objectives include using clinic visit data to identify opportunities for health interventions at participating schools and to characterize the type and number of student visits to the school nurses. An electronic module was developed in the State Electronic Notifiable Disease Surveillance System (SendSS) to facilitate data entry by participating school nurses and data management by the Georgia Department of Public Health.
\end{abstract}

\section{Introduction}

The Syndromic Surveillance Program (SSP) of the Georgia Department of Public Health collects chief complaint data from hospitals to characterize health trends in near real time. These data were critical for situational awareness during the 2009 H1N1 pandemic. In 2012, SSP and the Effingham County Schools began a project to collect syndromic surveillance data from school clinics. The hypothesis was that these data may be used to inform interventions during a pandemic, guide school health programs, elucidate health priorities in school-age populations, and quantify nursing staff needs in schools. Analysis of data from the first two pilot years has provided a novel look at the disparate burden of disease among students across schools in the county.

\section{Methods}

Every day during the 2012-2013 and 2013-2014 school years, 12 nurses from 12 schools in Effingham County entered data into a web-based module for clinic visits that met the following syndrome definitions: Influenza-Like Illness, Rash/Fever, Gastrointestinal, Injury, Asthma, Diabetes, and Total Daily Visits. At the end of the 2012-2013 school year, the school nurses were asked to review the syndromes. As a result, "Asthma" and "Diabetes" were modified to include "Acute Asthma" and "Acute Diabetes" counts. These new categories captured urgent visits while "Asthma" and "Diabetes" captured routine management. Additionally, "Oral Health" and "Mental Health" were added to the syndrome list. Given the modification of syndromes, for this abstract, only data from the 20132014 school year were analyzed using Microsoft Excel and SAS.

\section{Results}

During the 2013-2014 school year, Effingham County school nurses provided care for 79,906 total student visits; 24,301 (30.4\%) met at least one of the syndrome case definitions. Injury $(6,469$, $26.6 \%$ ) was the most common chief complaint, followed by diabetes management $(4,477,18.4 \%)$. Visits for asthma ranged from $10.6 \%$ of surveillance related visits in Elementary Schools to $2.5 \%$ of visits in High Schools. The proportion of visits related to asthma varied from $0.1 \%$ at one elementary school to $18.8 \%$ at another elementary school. Similarly, one middle school reported $21.3 \%$ of visits were related to diabetes, while the other two middle schools had less than $2 \%$ of visits related to diabetes.

\section{Conclusions}

This project demonstrated that school clinics are a significant resource for primary healthcare among school-aged children and that clinic syndromic surveillance is valuable for preparedness and education programs. Baseline burdens of both infectious and chronic diseases may be established, which can be used to identify trends or outbreaks in the future. These data also show that priority targets for school and community programs include injury prevention, asthma, and diabetes control. Evidence of poor chronic disease management among some students provided an opportunity for collaboration with chronic disease programs at DPH, perhaps leading to future funding for school-based intervention programs. Further analysis will be done to look at environmental factors and socioeconomic status of each school's population to determine the possible effect on disease burden and management of chronic disease. This information may be used to inform decisions for school inclusion in intervention programs.

\section{Keywords}

School Health; Syndromic Surveillance; State and Local Collaboration; Novel surveillance system; Student Health Surveillance

\section{Acknowledgments}

We would like to thank the school nurses and Marsha Cornell of Effingham County for their dedication and contribution to the success of this project.

\footnotetext{
*Amanda Feldpausch

E-mail: amanda.feldpausch@dph.ga.gov
} 